\title{
Generalized second law of thermodynamics in presence of interacting tachyonic field and scalar (phantom) field
}

\author{
Surajit Chattopadhyay ${ }^{1 *}$ and Ujjal Debnath ${ }^{2 \dagger}$ \\ ${ }^{1}$ Department of Computer Application, Pailan College of Management \\ and Technology, Bengal Pailan Park, Kolkata-700 104, India. \\ ${ }^{2}$ Department of Mathematics, Bengal Engineering and Science University, Shibpur, Howrah-711 103, India.
}

(Dated: November 7, 2018)

\begin{abstract}
In the present work, we have considered tachyonic field, phantom field and scalar field in both interacting and non-interacting situations and investigated the validity of the generalized second law of thermodynamics in a flat FRW universe. We found that in all cases, excepting the phantom field dominated universe, the derivative of the entropy is remaining at negative level and is increasing with the decrease in the redshift.
\end{abstract}

PACS numbers: 98.80.-k, 98.80.Cq, 95.36.+x

Since the discovery of the fact that presently there is an accelerating expansion of the universe; different suggestions have been made in order to explain the accelerated expansion. Among the various suggestions, one is the hypothesis of dark energy (DE), which is a fluid of negative pressure representing about $70 \%$ of the total energy of the universe $[1,2,3,4]$. The distinguishing feature of dark energy is that it violates the strong energy condition $\rho+3 p>0$ [5]. Strength of this acceleration depends on the theoretical model employed while interpreting the data. A wide range of scenarios have been proposed to explain this acceleration but most of them cannot explain all the features of the universe or they have so many parameters that they are difficult to fit. The models which have been discussed widely in literature are those which consider vacuum energy (cosmological constant) as DE, introduce a fifth element and dub it quintessence $(\omega>-1)$ or scenarios named phantom with $\omega<-1$, where $\omega=p / \rho$ is a parameter of state [6].

One remarkable feature of the phantom model is that the universe will end with a "big rip" (future singularity). That is, for phantom dominated universe, its total lifetime is finite. Before the death of the universe, the phantom dark energy will rip apart all bound structures like the Milky Way, solar system, Earth and ultimately the molecules, atoms, nuclei and nucleons of which we are composed. In the quintessence model, the equation state $\omega=p / \rho$ is always in the range $-1 \leq \omega \leq 1$ for the potential $V(\phi)>0$. It has been shown in the reference [7] that the decay of an unstable D-brane produces pressure-less gas with finite energy density that resembles classical dust. The cosmological effects of the tachyon rolling down to its ground state have been discussed by the reference [8]. There have been many works aimed at connecting the string theory with inflation. While doing so, various ideas in string theory based on the concept of branes have proved themselves fruitful. Rolling tachyon matter associated with unstable D-branes has an interesting equation of state which smoothly interpolates between -1 and 0 . As the Tachyon field rolls down the hill, the universe experiences accelerated expansion and at a particular epoch the scale factor passes through the point of inflection marking the end of inflation [9].

To obtain a suitable evolution of the Universe an interaction is often assumed such that the decay rate should be proportional to the present value of the Hubble parameter for good fit to the expansion history of the Universe as determined by the Supernovae and CMB data [10]. These kinds of models describe an energy flow between the components so that no components are conserved separately. There are several works on the interaction between dark energy (tachyon or phantom) and dark matter $[11,12,13,14]$, where phenomenologically introduced different forms of interaction term.

Izquierdo and Pavon [15] explored the thermodynamic consequences of phantom-dominated universe and mentioned that one must take into account that ever accelerating universe has a future event horizon (or cosmological horizon). Since the horizon implies a classically unsurmountable barrier to our ability to see what lies beyond it, it is natural to attach an entropy to the horizon size (i.e., to its area) for it is a measure of our

\footnotetext{
*surajit_2008@yahoo.co.in

† ujjaldebnath@yahoo.com, ujjal@iucaa.ernet.in
} 
ignorance about what is going on in the other side [16]. Setare [16] investigated the validity of the generalized second law of thermodynamics for the quintom model of dark energy. Setare and Shafei [17] employed a holographic model of dark energy to investigate the validity of the first and second laws of thermodynamics in a non-flat (closed) universe. Setare [18] considered the interacting holographic model of dark energy to investigate the validity of the generalized second law of thermodynamics in a non-flat (closed) universe enclosed by the event horizon. The present work is different from the works mentioned above. In the present work, we have investigated the validity of the second law of thermodynamics in the presence of interacting tachyonic field and scalar (phantom) field in a spatially flat isotropic and homogeneous FRW universe whose metric is given by

$$
d s^{2}=d t^{2}-a^{2}(t)\left[d r^{2}+r^{2}\left(d \theta^{2}+\sin ^{2} \theta d \phi^{2}\right)\right]
$$

where $a(t)$ is the scale factor. we consider a two fluid model consisting of tachyonic field and scalar field (or phantom field).

Before going into the technical details we discuss the motivation behind this study. In various previous works, the present accelerated expansion of the universe has been studied by means of single components of dark energy. Sami et al [19] examined the possibility of rolling tachyon to play the dual role of inflaton at early epochs and dark matter at late times. Chattopadhyay et al [20] studied the acceleration of the universe in presence of tachyonic field and showed that it interpolates between dust and $\Lambda$ CDM. Sing et al [21] studied the general features of the dynamics of the phantom field in the cosmological context. In the case of inverse coshyperbolic potential, they [21] demonstrated that the phantom field can successfully drive the observed current accelerated expansion of the universe with the equation of state parameter $\omega<-1$.

In the recent past, interacting dark energy candidates has been studied by various authors. Cai and Wang [12] studied a cosmological model in which phantom dark energy is coupled to dark matter by phenomenologically introducing a coupled term to the equations of motion of dark energy and dark matter. Macorra [22] studied the cosmological evolution of two coupled scalar fields with an arbitrary interaction term and discussed the possibility of having one of the scalar fields as of dark energy while the other could be a scalar field redshifting as matter. An interaction between phantom field and tachyonic field was considered by Chattopadhyay and Debnath [23]. Motivated by the investigations on the validity of generalized second law of thermodynamics in presence of phantom field [15], holographic dark energy [17], quintom [16] and surveying the literatures on two-component models $[12,18,22,23,24,25]$ we decided to investigate the validity of the generalized second law of thermodynamics in presence of interacting as well as non-interacting tachyonic field and scalar (phantom) field.

As we are considering a two-component model, the total energy density and pressure are respectively given by

$$
\rho_{t o t}=\rho_{1}+\rho_{2}
$$

and

$$
p_{\text {tot }}=p_{1}+p_{2}
$$

The energy density $\rho_{1}$ and pressure $p_{1}$ for tachyonic field $\phi_{1}$ with potential $V_{1}\left(\phi_{1}\right)$ are respectively given by $[23]$

$$
\rho_{1}=\frac{V_{1}\left(\phi_{1}\right)}{\sqrt{1-\dot{\phi}_{1}^{2}}}
$$

and

$$
p_{1}=-V_{1}\left(\phi_{1}\right) \sqrt{1-\dot{\phi}_{1}^{2}}
$$

The energy density $\rho_{2}$ and pressure $p_{2}$ for scalar field (or phantom field) $\phi_{2}$ with potential $V_{2}\left(\phi_{2}\right)$ are respectively given by $[23]$ 


$$
\rho_{2}=\frac{\epsilon}{2} \dot{\phi}_{2}^{2}+V_{2}\left(\phi_{2}\right)
$$

and

$$
p_{2}=\frac{\epsilon}{2} \dot{\phi}_{2}^{2}-V_{2}\left(\phi_{2}\right)
$$

where, $\epsilon=1$ for scalar field and $\epsilon=-1$ for phantom field.

Therefore, the conservation equation reduces to

$$
\dot{\rho}_{1}+3 H\left(\rho_{1}+p_{1}\right)=-Q
$$

and

$$
\dot{\rho}_{2}+3 H\left(\rho_{2}+p_{2}\right)=Q
$$

Here, $Q$ is the interaction term. If $Q$ is positive, then there will be a transfer of energy from $\rho_{1}$ to $\rho_{2}$ [24]. For solving the above equations different forms for the interaction term $Q$ are considered. For getting convenience while integrating equation (8), we have chosen $Q=3 \delta H \rho_{1}$ where $\delta$ is the interaction parameter and $H=\dot{a}(t) / a(t)$. Similar choice of interaction term has been made in [26]. A detailed discussion on $Q$ is available in $[25]$.

In an earlier work by the authors of the present work (Reference [23]), the solutions for $\dot{\phi}_{1}, \dot{\phi}_{2}, V_{1}$, and $V_{2}$ were obtained under interaction as follows:

$$
\begin{aligned}
& \dot{\phi}_{1}^{2}=\left[-\delta+\left(\frac{c}{a^{3}}\right)^{\frac{2(1+\delta)}{1+2 m}}\right]\left[1+\left(\frac{c}{a^{3}}\right)^{\frac{2(1+\delta)}{1+2 m}}\right]^{-1} \\
& \dot{\phi}_{2}^{2}=-\frac{2 \dot{H}}{\epsilon}+\frac{1}{\epsilon}\left[1+\left(\frac{c}{a^{3}}\right)^{\frac{2(1+\delta)}{1+2 m}}\right]^{m-\frac{1}{2}}\left[\delta-\left(\frac{c}{a^{3}}\right)^{\frac{2(1+\delta)}{1+2 m}}\right](1+\delta)^{-m-\frac{1}{2}} \\
& V_{1}=\left[1+\left(\frac{c}{a^{3}}\right)^{\frac{2(1+\delta)}{1+2 m}}\right]^{m}(1+\delta)^{-m} \\
& V_{2}=\dot{H}+3 H^{2}-\frac{1}{2}\left[1+\left(\frac{c}{a^{3}}\right)^{\frac{2(1+\delta)}{1+2 m}}\right]^{m-\frac{1}{2}}\left[2+\delta+\left(\frac{c}{a^{3}}\right)^{\frac{2(1+\delta)}{1+2 m}}\right](1+\delta)^{-m-\frac{1}{2}}
\end{aligned}
$$

where, $c$ is an integration constant, $m>0, n>0$, and it was assumed that $V_{1}=\left(1-\dot{\phi}_{1}^{2}\right)^{-m}$, and $V_{2}=n \dot{\phi}_{2}^{2}$.

In the present work, we consider the universe as a flat FRW universe and take into account that the accelerating universe has a future event horizon $R_{h}$, which is also named as cosmological horizon. The radius of observer's event horizon is given by $[16,27]$

$$
R_{h}=a \int_{t}^{\infty} \frac{d t}{a}=a \int_{a}^{\infty} \frac{d a}{H a^{2}}
$$

To study the generalized second law (GSL) through the universe under the interaction between tachyonic field and scalar (phantom) field we would examine the nature of the derivative of the normal entropy $S$ in presence of interaction. It is a proven fact that for phantom dominated universe $\dot{S}>0$ and for a quintessence 
dominated universe $\dot{S}<0$ [16]. Our target is to answer the question : Is $\dot{S}>0$ under the interaction between tachyonic field and scalar (phantom) field?.

We consider the FRW universe as a thermodynamical system with the horizon surface as a boundary of the system. In general, the radius of the event horizon $R_{h}$ is not constant but changes with time. Let $d R_{h}$ be an infinitesimal change of the event horizon radius during a time of interval $d t$. This small displacement $d R_{h}$ will cause a small change $d V$ in the volume $V$ of the event horizon. Each spacetime describing a thermodynamical system and satisfying Einsteins equations differs infinitesimally in the extensive variables volume, energy and entropy by $d V, d E$ and $d S$, respectively, while having the same values for the intensive variables temperature $T$ and pressure $P$ [18]. Thus, for these two spacetimes describing two thermodynamical states, there must exist some relation among these thermodynamic quantities. It turns out that the differential form of the Friedman equation can be rewritten as a universal form $[16,17]$

$$
T d S=d E+p d V=(p+\rho) d V+V d \rho
$$

Also we know that

$$
H^{2}=\frac{1}{3} \rho
$$

and

$$
\dot{H}=-\frac{1}{2}(p+\rho)
$$

Using $V=\frac{4}{3} \pi R_{h}^{3}$ in equation (15) we get

$$
T d S=-2 \dot{H} d V+V d \rho=-8 \pi R_{h}^{2} \dot{H} d R_{h}+8 \pi R_{h}^{3} d H
$$

From equation (18), it can be obtained that

$$
\dot{S}=\frac{8 \pi \dot{H} R_{h}^{2}}{T}
$$

If the horizon entropy is taken to be $S_{h}=\pi R_{h}^{2}[16]$, we get

$$
\dot{S}+\dot{S}_{h}=\frac{8 \pi \dot{H} R_{h}^{2}}{T}+2 \pi R_{h} \dot{R}_{h}
$$

Taking the temperature $T=\frac{1}{2 \pi R_{h}}$ and using $\dot{H} R_{h}=H R_{h}-1[16]$ we can write

$$
\dot{S}+\dot{S}_{h}=\dot{S}_{X}=16 \pi^{2} \dot{H} R_{h}^{3}+2 \pi R_{h}\left(H R_{h}-1\right)
$$

At this stage it should be stated that the thermal equilibrium of the different components with one another and/or with the horizon cannot be assured. In this work it is acknowledged that the thermal equilibrium is just an additional hypothesis.

In the present problem, while considering tachyonic field, scalar field and phantom field separately, the suffix $X$ of equation (21) would be replaced by tachyon, scalar, and phantom respectively. While considering the mixture of tachyon and scalar (phantom) fields as a mixture without interaction (i.e. $\delta=0$ ), $X$ would be replaced by mixture and in the interacting case (i.e. $\delta \neq 0$ ), $X$ would be replaced by total.

Equations (16) and (17) are now implemented with the pressure and energy densities corresponding to the cases mentioned in the last paragraph. Subsequently, $R_{h}$ could be obtained in all the cases. Furthermore, $\dot{S}_{X}$ 


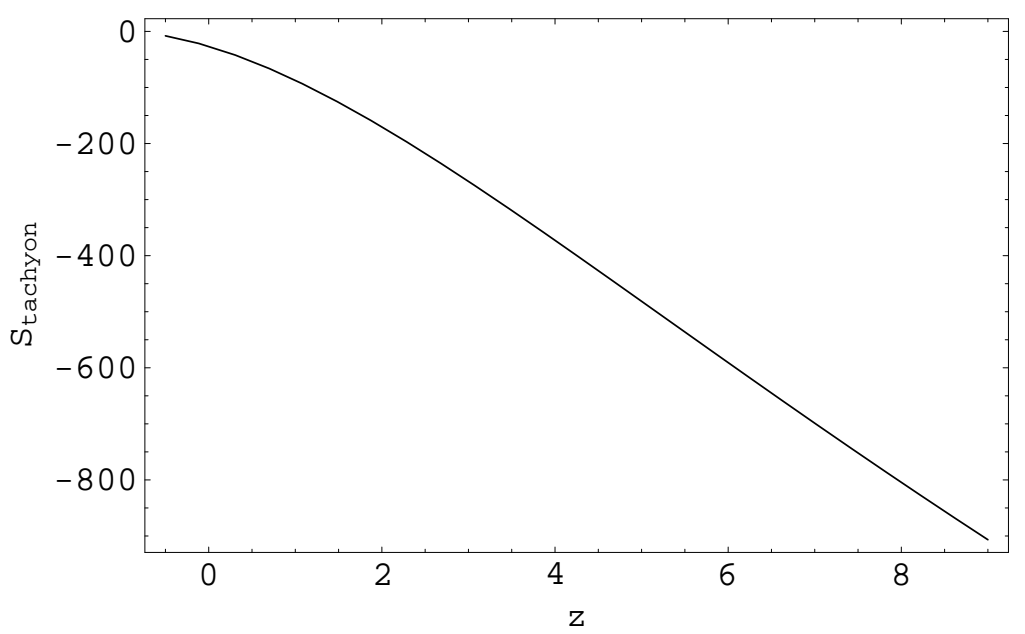

Fig.1 shows the plot of $\dot{S}_{\text {tachyon }}$ against $z$ when only tachyonic field is considered. The vertical axis represents

the time derivative of entropy.

was obtained based on (16) and (17). The $\dot{S}_{X}$ are plotted against the redshift $z=1-a^{-1}$.

Now we investigate the GSL of thermodynamics when the universe is filled with (i) only tachyonic field, (ii) only phantom field, (iii) only normal scalar field, (iv) mixture of tachyon and scalar (phantom) fields without interaction and (v) mixture of tachyon and scalar (phantom) fields with interaction.

\section{Case I: GSL in presence of only tachyonic field}

We consider equations (4) and (5) where the potential is obtained from equation (12) with $\delta=0$. Subsequently $\dot{S}_{\text {tachyon }}$ is plotted against redshift $z$ in figure 1 . It is observed from the said figure that $\dot{S}_{\text {tachyon }}$ has an increasing nature with decrease in $z$. In this figure is it also visible that $\dot{S}$ is remaining negative when only tachyonic field is considered. At the same time we have noted that $R_{h}$ always remains at positive level.

\section{Case II: GSL in presence of only phantom field}

We consider equations (6) and (7) with $\epsilon=-1$ and the potential is obtained from equation (13) with $\delta=0$. Subsequently $\dot{S}_{\text {phantom }}$ is plotted against redshift $z$ in figure 2. It is observed from the said figure that $\dot{S}_{\text {phantom }}$ has a decreasing nature with decrease in $z$. In this figure is it also apparent that $\dot{S}$ is remaining positive when only phantom field is considered. At the same time we have noted that $R_{h}$ always remains at positive level.

\section{Case III: GSL in presence of only scalar field}

We consider equations (6) and (7) with $\epsilon=1$ and the potential is obtained from equation (13) with $\delta=0$. Subsequently $\dot{S}_{\text {scalar }}$ is plotted against redshift $z$ in figure 3 . It is observed from the said figure that $\dot{S}_{\text {scalar }}$ has a decreasing nature with decrease in $z$. In this figure is it also apparent that $\dot{S}$ is remaining negative when only scalar field is considered. At the same time we have noted that $R_{h}$ always remains at positive level.

\section{Case IV: GSL in presence of a mixture of tachyonic field and scalar (phantom) field without interaction}

Equations (2) and (3) are used to calculate the energy density and pressure of the two-component system of tachyonic field and scalar (phantom) field. $\rho_{1}, \rho_{2}, p_{1}$, and $p_{2}$ are computed using (4)-(7), and (10)-(13). However, the non-interacting situation being considered, we put $\delta=0$. Figures 4 and 5 display the evolution of $\dot{S}_{\text {total }}$ in the cases of tachyonic field mixing with phantom and scalar field respectively. Both of the figures display almost similar pattern of evolution of $\dot{S}_{\text {total }}$ with redshift $z$. In both of the cases the derivative of the 


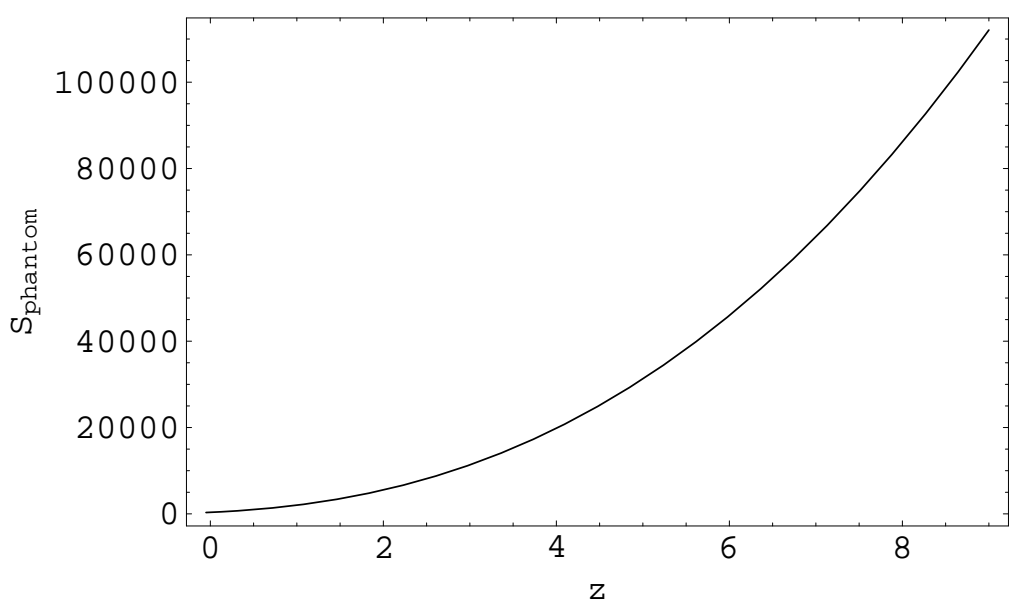

Fig.2 plots $\dot{S}_{\text {phantom }}$ against $z$ when only phantom field is considered. The time derivative of entropy is presented along the vertical axis.

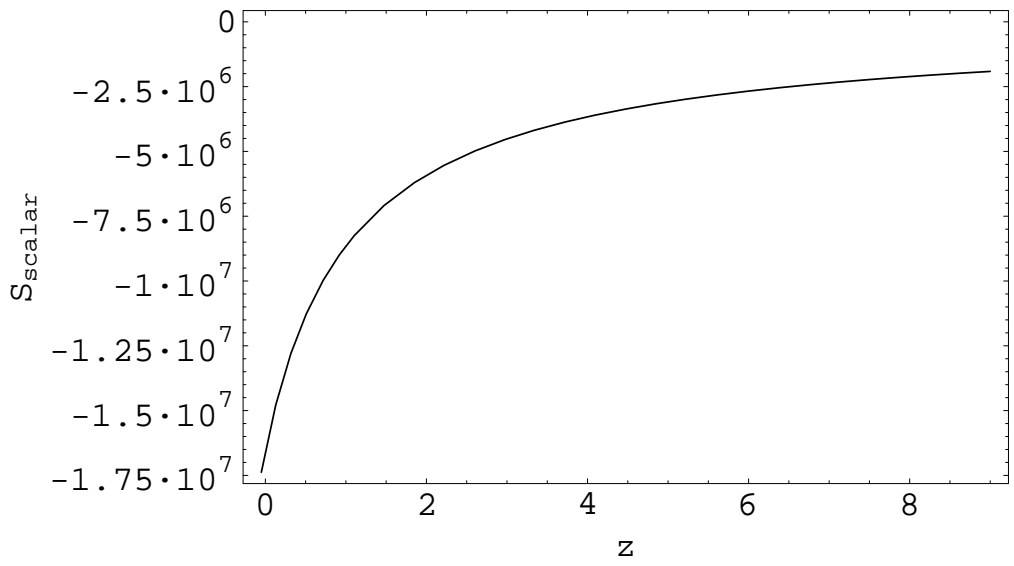

Fig.3 plots $\dot{S}_{\text {scalar }}$ against $z$ when only scalar field is considered. The time derivative of entropy is presented along the vertical axis.

entropy is staying at negative level and is increasing with decrease in the redshift. In both of the cases we have seen that the event horizon $R_{h}$ is remaining positive.

\section{Case V: GSL in presence of interaction between tachyonic field and scalar (phantom) field}

In the present case, we consider the interacting situation. Therefore, in the conservation equation (8), the interaction parameter $\delta$ is kept at non-zero level. Equations (2) and (3) are used to calculate the energy density and pressure of the two-component system of tachyonic field and scalar (phantom) field. $\rho_{1}, \rho_{2}, p_{1}$, and $p_{2}$ are computed using (4)-(7), and (10)-(13). Figures 6 and 7 display the evolution of $\dot{S}_{\text {total }}$ in the cases of tachyonic field interacting with phantom and scalar field respectively. It is found that the figures 6 and 7 are displaying similar pattern. That is, in presence of interaction, the variation of $\dot{S}_{\text {total }}$ with decrease in redshift is almost same i.e. increasing. Moreover, the pattern has similarity with the non-interacting cases too. Here 


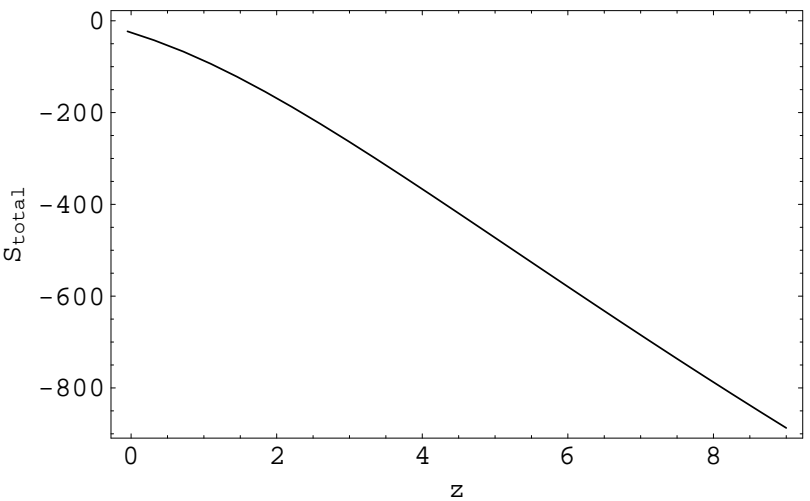

Fig.4

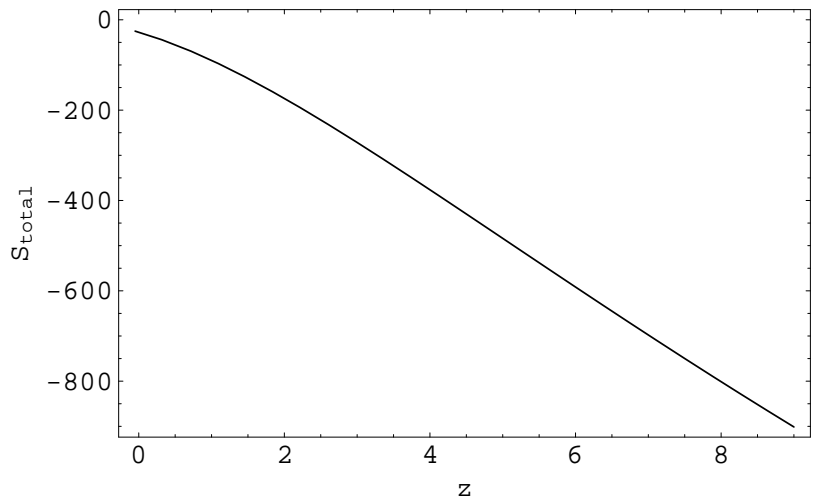

Fig.5

Figs. 4 and 5 plot $\dot{S}_{\text {total }}$ against $z$ when a tachyonic field has mixed with phantom field and scalar field respectively without interaction $(\delta=0)$. The time derivative of entropy is presented along the vertical axes.

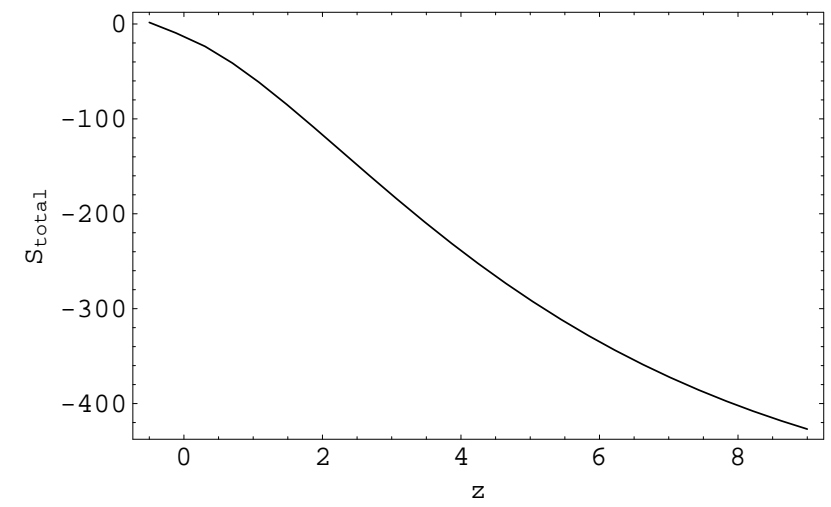

Fig.6

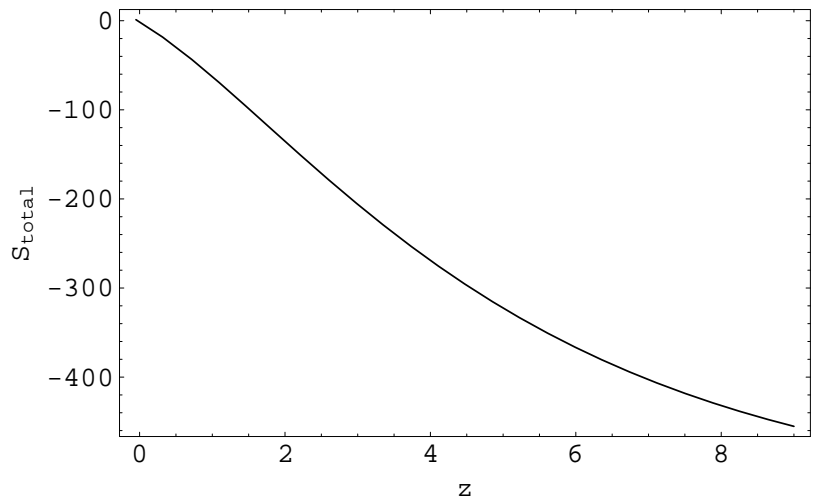

Fig.7

Figs. 6 and 7 show the plot of $\dot{S}_{\text {total }}$ against $z$ when tachyonic field is interacting with phantom and scalar field respectively $(\delta=0.03)$. The time derivative of entropy is presented along the vertical axis.

also, the derivative of the entropy is remaining at negative level. We have tested that $R_{h}$ is keeping positive with decrease in the redshift. The figures display the plotting with $\delta=0.03$. Despite varying the values of $\delta$ to other positive and negative values the pattern has remained the same.

In this work, we fixed our target at answering the question: $I s \dot{S}>0$ under the interaction between tachyonic field and scalar (phantom) field? In the previous paragraphs, we have searched for the answer to this question. While doing so we have investigated several competitive cases. The candidates of dark energy, namely, tachyonic field, phantom field, and scalar field have been considered in this study. Investigating the second law of thermodynamics, the globally accepted principle in the universe, both single component and two-component models (interacting and non-interacting) have been considered. In the flat FRW universe, we have seen that in all the above cases the event horizon $R_{h}$ is staying at positive level. However, the derivative of the entropy $\dot{S}_{X}=\dot{S}+\dot{S}_{h}$ has displayed varied natures in the cases under consideration. When we considered a flat FRW universe dominated by tachyonic field only, we found that the derivative of the entropy is increasing with decrease in the redshift. However, in phantom dominated and scalar field dominated cases, it is decreasing with decrease in the redshift. Another notable thing is that, excepting the phantom dominated universe, the derivative of the entropy is remaining at the negative level. We further noted that, the increase in the 
derivative of the entropy in the case of tachyonic field dominated universe is more sharp than the decrease in the derivative of the entropy with decrease in the redshift in the other two cases. We have further seen that if we consider the two-component model, then also the evolution of the derivative of the entropy with decrease in the redshift is almost similar to the tachyonic field dominated universe. Even the introduction of interaction parameter $\delta$ at positive or negative level does not affect this pattern significantly. In these cases too, the derivative of the entropy remains negative.

\section{Acknowledgement:}

The authors wish to acknowledge the warm hospitality provided by the Inter University Centre for Astronomy and Astrophysics (IUCAA), Pune, India, where the work was carried out during a scientific visit in January, 2010. The authors are thankful to the anonymous reviewers for giving constructive comments to enhance the quality of the work.

\section{References:}

[1] E. J. Copeland, M. Sami and S. Tsujikwa, IJMPD 15, 1753 (2006).

[2]A. G. Riess et al., Astrophys. J. 116, 1009 (1998).

[3] A. G. Riess et al., Astron. J. 117, 707 (1999).

[4] Y.-F. Cai, E. N. Saridakis, M. R. Setare and J.-Q. Xia, Phys. Rept. 493, 1 (2010).

[5] S. J. Perlmutter et al, Astrophys. J. 517, 565 (1999).

[6] R. R. Caldwell, Phys. Lett. B 545, 23 (2002).

[7] A. Sen, JHEP 0204048 (2002); JHEP 0207, 065 (2002).

[8] G. W. Gibbons, Phys. Lett. B 537, 1 (2002).

[9] M. Sami, Mod. Phys. Lett. A 18, 691 (2003).

[10] M. S. Berger and H. Shojaei, Phys. Rev. D 74, 043530 (2006).

[11] R. Herrera, D. Pavon and W. Zimdahl, Gen. Rel. Grav. 36, 2161 (2004).

[12] R. -G. Cai and A. Wang, JCAP 0503, 002 (2005).

[13] Z. -K. Guo, R. -G. Cai and Y. -Z. Zhang, JCAP 0505, 002 (2005).

[14] T. Gonzalez and I. Quiros, Class. Quantum Grav. 25, 175019 (2008).

[15] G. Izquierdo and D. Pavon, Phys. Lett. B 633, 420 (2006).

[16] M. R. Setare, Phys. Lett. B 641, 130 (2006).

[17] M. R. Setare and S. Shafei, JCAP 09, 011 (2006)

[18] M. R. Setare, JCAP 01, 023 (2007).

[19] M. Sami, P. Chingangbam and T. Qureshi, Pramana 62, 765 (2004).

[20] S. Chattopadhyay, U. Debnath and G. Chattopadhyay, Astrophys. Space Sci. 314, 41 (2008).

[21] P. Singh, M. Sami and N. Dadhich, Phys. Rev. D 68, 023522 (2003).

[22] A. de la Macorra, JCAP 030 (2008).

[23] S. Chattopadhyay and U. Debnath, Brazilian J. Phys. 39, 86 (2009).

[24] M. Cataldo, P. Mella, P. Minning and J. Saavedra, Phys. Lett. B 662, 314 (2008).

[25] M. R. Setare, J. Sadeghi and A. R. Amani, Phys. Lett. B 673, 241 (2009).

[26] B. Wang, Y. Gong and E. Abdalla, Phys. Lett. B 624, 141 (2005).

[27] M. R. Setare, Eur. Phys. J. C 50, 991 (2007). 